\title{
Erratum to: Drivers of Inclusive Innovation in Developing Country Markets: A Policy Perspective
}

\author{
Christopher Foster • Richard Heeks
}

\section{Erratum to:}

The book was inadvertently published with wrong chapter title for chapter 4; the correct title should read as below:

Drivers of Inclusive Innovation in Developing Country Markets: A Policy Perspective

The updated original online version for this chapter can be found at http://dx.doi.org/10.1057/978-1-137-60168-1_4 